\title{
Delay and Capacity in Energy Efficient Sensor Networks
}

\author{
Nabhendra Bisnik \\ ECSE Department \\ Rensselaer Polytechnic Institute \\ Troy, NY 12180 \\ bisnin@rpi.edu
}

\author{
Alhussein A. Abouzeid \\ ECSE Department \\ Rensselaer Polytechnic Institute \\ Troy, NY 12180 \\ abouzeid@ecse.rpi.edu
}

\begin{abstract}
MAC protocols for wireless sensor networks employ periodic switching to low energy sleep state in order to enhance network lifetime. During the sleep state, the sensors do not perform energy consuming operations such as receiving and transmitting packets. During the normal state, CSMA based multi-access mechanism is the MAC protocol of choice in distributed, unsynchronized sensor networks. The energy conserving mechanism has a two-fold effect on delay in the network. On one hand it increases delay since many a times the intended receiver may be in sleep state and the transmitter has to delay the transmission to allow the receiver to wake up. On the other hand, since the sensors do not transmit in sleep state, the contention for channel is reduced which tends to improve delay. In this paper we present a queuing theoretic analysis of delay and capacity in sensor networks with uncoordinated sleep mechanism and characterize the energy-delay-capacity tradeoffs. We consider several sleep states which consume different levels of energy. We model sensor networks as queuing networks and evaluate closed form expressions for average packet delay and maximum achievable pernode throughput in terms of network parameters and sleep schedule. Comparisons with the performance of networks that do not employ any energy conserving mechanisms show that any of the energy conserving sleep states in the networks considered in this paper leads to considerable degradation in delay and capacity of the network.
\end{abstract}

\section{Categories and Subject Descriptors}

C.2 [Computer Systems Organization]: Computer Communication Networks; C.4 [Computer Systems Organization]: Performance of Systems; G.0 [Mathematics of Computing]: General; I.6 [Computing Methodologies]: Simulation and Modeling

\section{General Terms}

Theory, Performance

\section{Keywords}

Queuing Theory, Delay, Throughput, Sensor Networks

Permission to make digital or hard copies of all or part of this work for personal or classroom use is granted without fee provided that copies are not made or distributed for profit or commercial advantage and that copies bear this notice and the full citation on the first page. To copy otherwise, to republish, to post on servers or to redistribute to lists, requires prior specific permission and/or a fee.

PE-WASUN'07, October 22, 2007, Chania, Crete Island, Greece. Copyright 2007 ACM 978-1-59593-808-4/07/0010 ...\$5.00.

\section{INTRODUCTION}

Battery power is a very important resource in sensor networks. This is particularly true in sensor networks that are deployed in areas of poor accessibility. These sensor networks are expected to operate for long periods without any human intervention, ruling out the possibility of replacing exhausted batteries. Such sensors have to be highly energy efficient in order to conserve battery power. Among other schemes, such as choosing energy efficient routes $[1,2]$, scheduling [3-5], data aggregation [6], etc, sensors endeavor to conserve energy by periodically switching to a low energy sleep state [7-9]. When a node is not in sleep state, it is in a state termed active state. Since most of the energy is consumed in packet transmission and idle listening, the sensors do not perform these operations during the sleep state. However, other low energy operations, such as sensing and data processing, may be allowed during the sleep state. In a dense sensor network, where there are a lot of redundant sensors, the overall operation of the sensor network may not suffer even if the sensors do not sense or receive packets during the sleep state. This is particularly true if the sleep schedules of the sensors are uncorrelated. We refer to such sensor networks where the nodes switch to low energy sleep state as energy efficient sensor networks.

Other than enhancing the network lifetime, the periodic switch to sleep state has a two-fold effect on the delay and capacity of an energy efficient network. On the positive side, since the nodes do not transmit packets during sleep state, the number of nodes contending for the wireless channel is reduced. This reduces the time a node has to wait to transmit a packet and hence tends to improve the delay and capacity of the network. However, it also tends to have a negative effect on delay and capacity. There are two reasons for this. Firstly, sleep state prohibits a node from transmission and hence does not let it utilize the channel even when it may be idle. Secondly, even though a node may be in active state and ready to transmit a packet, the node that is supposed to be the next hop of the packet may be in sleep state. This effect of the periodic switch to sleep state tends to increase the delay experienced by a packet and reduces the capacity of the network.

In this paper we explore in detail the effect of the sleep mechanism in energy efficient sensor networks on the delay and capacity of the network. We use a queuing theoretic approach, similar to the one used in [10]. The study is mainly targeted towards battery powered sensor networks, although the results may apply to other battery powered ad hoc networks that use energy efficient MAC. We consider various sleep states, differentiated on the basis of whether packet receive and/or sense operation is allowed in the sleep state. The network model takes into account the packet generation process, degree of locality of traffic, and the random access MAC mechanism for packet transmission during the active state. 
We develop a G/G/1 queuing network model for the energy efficient sensor networks and use diffusion approximation in order to solve the queuing network. We evaluate closed form expressions for the maximum allowable sensing rate and the average end-to-end packet delay in energy efficient sensor networks.

The degradation of delay performance due to MAC protocols with sleep has been the focus of several recent studies. In [11], the authors evaluate an expression for average delay in a one-hop network that employs a random access MAC with sleep. Percolation based approach is used in [12] to analyze bounds on delay incurred in reporting a sensed event to a sink of a dense sensor network. It is shown that the delay in relaying a packet from a source to its destination is proportional to the distance between the two nodes. The value of the proportionality constant however depends on network parameters such as node density, communication radius, sleep schedule, etc. Also it is shown that the size of the set of nodes that may receive a broadcast message from a source grows linearly with time. However, the effect of nodes competing for the wireless channel is not considered in this work. The effect of sporadic and correlated traffic generation in one-hop sensor networks on delay is considered in [13]. The problem of finding sleep schedules that improve the delay performance is considered in [14-16]. In [14], the authors propose DMAC that minimizes sleep latency in scenarios where the communication is restricted to unidirectional data gathering trees. A graph theoretic approach is used to formulate the optimal sleep schedule problem for arbitrary communication pattern. It is shown that the problem is NP-hard and heuristic algorithms are proposed to improve the delay performance. A protocol for sleep scheduling is proposed in [16] that minimizes the average delay between occurrence of an event and its detection by a sensor.

In our work we consider a multi-hop sensor network where CSMA is used as the multi-access protocol for packet transmission. The communication considered in this paper corresponds to many-tomany communication pattern. Uncoordinated sleep schedule is considered, which is the least complex scheduling policy in large distributed sensor networks. Thus our analysis takes into account both effects of sleep - latency because of unavailability of next hop node and decrease in contention due to reduction in number of nodes that contend for the channel. We evaluate closed form expressions for end-to-end delay and maximum achievable throughput for various sleep scenarios. Our results indicate that no matter what operations are permitted during sleep state, the performance of the network always degrades whenever any energy saving mechanism is used. Depending upon the goals of the applications, the analytical results may be used to select the appropriate parameters of sleep schedule to configure the network.

The rest of the paper is organized in the following manner. We briefly present the diffusion approximation method in Section 2 . The network model is described in Section 3. The delay analysis for energy efficient networks is presented in Section 4. The results are discussed in detail in Section 5. We finally summarize the paper in Section 6.

\section{DIFFUSION APPROXIMATION METHOD}

The diffusion approximation [17] can be used for solving an open G/G/1 queuing network provided that all the nodes in the network are single server with first-come first-serve (FCFS) service strategy. The advantage of using the diffusion approximation in this work is that it allows us to obtain closed form expressions for the average end-to-end delay.

In this section we briefly describe how the diffusion approximation is used to solve an open G/G/1 queuing network. (Please see [17] for details). Suppose we have an open queuing network with $n$ service stations, numbered from 1 to $n$. The external arrival of a job is a renewal process with an average inter-arrival time of $1 / \lambda_{e}$ and the coefficient of variance of inter-arrival time equals $c_{A}$. The mean and coefficient of variance of the service time at a station $i$ are denoted by $1 / \mu_{i}$ and $c_{B i}$, respectively.

The visit ratio of a station in a queuing network is defined as the average number of times a packet is forwarded by (i.e. visits) the station. The visit ratio of station $i$, denoted by $e_{i}$, is given by

$$
e_{i}=p_{0 i}(n)+\sum_{j=1}^{j=n} p_{j i}(n) \cdot e_{j}
$$

where $p_{0 i}$ denotes the probability that a job enters the queuing network from station $i$ and $p_{j i}$ denotes the probability that a job is routed to station $i$ after completing its service at station $j$.

There are two sources of packet arrivals at a station: the jobs that are generated at the station and the jobs that are forwarded to the station by other stations. The resulting arrival rate is termed the effective arrival rate at a station. The effective arrival rate at the station $i$, denoted by $\lambda_{i}$ is given by

$$
\lambda_{i}=\lambda_{e} e_{i}
$$

The utilization factor of station $i$, denoted by $\rho_{i}$, is given by

$$
\rho_{i}=\lambda_{i} / \mu_{i}
$$

The squared coefficient of variance of the inter-arrival time at a station $i$, denoted by $c_{A i}^{2}$, is approximated using

$$
c_{A i}^{2}=1+\sum_{j=0}^{n}\left(c_{B j}^{2}-1\right) \cdot p_{j i}^{2} \cdot e_{j} \cdot e_{i}^{-1}
$$

where $c_{B 0}^{2}=c_{A}^{2}$

According to the diffusion approximation, the approximate expression for the probability that the number of jobs at station $i$ equals $k$, denoted by $\hat{\pi}_{i}(k)$, is

$$
\hat{\pi}_{i}(k)= \begin{cases}1-\rho_{i} & \mathrm{k}=0 \\ \rho_{i}\left(1-\hat{\rho}_{i}\right) \hat{\rho}_{i}^{k-1} & \mathrm{k}_{i} 0\end{cases}
$$

where

$$
\hat{\rho}_{i}=\exp \left(-\frac{2\left(1-\rho_{i}\right)}{c_{A i}^{2} \cdot \rho_{i}+c_{B i}^{2}}\right)
$$

The mean number of jobs at a station $i$, denoted by $\overline{K_{i}}$, is

$$
\overline{K_{i}}=\rho_{i} /\left(1-\hat{\rho}_{i}\right)
$$

\section{QUEUING NETWORK MODEL}

In this section we present the network model and develop a queuing network model for multihop wireless networks. We also derive expressions for the parameters of the queuing network model.

\subsection{The network model}

Sensor placement and communication model: The network consists of $n+1$ sensor nodes, numbered 1 to $n+1$, that are distributed uniformly and independently over a torus of unit area. We assume a torus area in order to avoid complications in the analysis caused by the edge effects. Each node is assumed to have an equal transmission range, denoted by $r(n)$. Let $r_{i j}$ denote the distance between nodes $i$ and $j$. Nodes $i$ and $j$ are said to be neighbors if they can directly communicate with each other, i.e. if $r_{i j} \leq r(n)$. Let $N(i)$ denote the set of nodes that are neighbors of node $i$. All the neighbors of a node lie on a disc of area $A(n)=\pi r(n)^{2}$ centered at the node. The area $A(n)$ is termed the "communication 


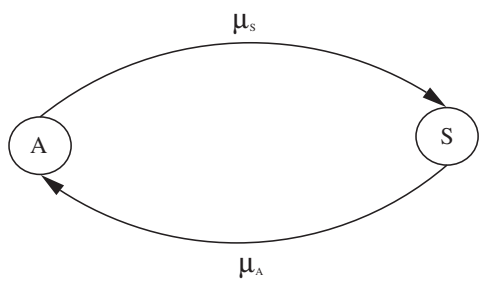

A : Active State

S : Sleep State

Figure 1: States of a node in an energy efficient sensor network.

area" of a node. The communication area is chosen such that the network is connected which ensures that $N(i) \neq \phi \forall i$. The transmission rate of each node equals $W$ bits/second.

We use a special case of the Protocol Model of interference described in [18]. If node $i$ transmits to node $j$ then the transmission will be successful only if (i) $r_{i j} \leq r(n)$ and (ii) $r_{k j}>r(n)$ for every other node $k \neq i, j$ that transmits simultaneously with node $i$. In other words, node $i$ can successfully transmit a packet to node $j$ only if $i$ is a neighbor of $j$ and no other neighbor of $j$ is transmitting concurrently with $i$. (This is equivalent to setting $\Delta=0$ in the Protocol Model in [18]).

Traffic model: Each node in the network could be a source, destination and/or relay of packets. Each node generates packets with rate $\lambda$ packets/sec. The delay analysis is possible for any packet generation process as long as the mean and SCV of packet inter arrival time are known. For the sake of simplicity, we assume in our model that the packet generation process at each node is an i.i.d. Poisson process. The size of each packet is constant and equals $L$ bits. When a node receives a packet from any of its neighbors, it either forwards the packet to its neighbors with probability $(1-p(n))$ or absorbs the packet with probability $p(n)$. The probability $p(n)$ is termed "absorption probability". In other words, the absorption probability is the probability that a node is the destination of a packet given that the node has received the packet from its neighbors. When a node forwards a packet, each of its neighbors is equally likely to receive the packet. The advantage of such a model is that we can control the locality of the traffic by varying the parameter $p(n)$. The traffic is highly localized if $p(n)$ is large while a small value of $p(n)$ implies unlocalized traffic. This would help us to characterize the effect of the locality of the traffic on the average delay and maximum achievable throughput. In addition, random walk had been proposed as a possible way to route packets $[19,20]$ and query packets [21-24] in wireless sensor networks.

Sleep model: We use the following sleep model for the analysis. Each sensor node is either in active or sleep state. The time spent by a node in the active state is exponentially distributed with mean $\frac{1}{\mu_{S}}$ while the time spent by a node in the sleep state is exponentially distributed with mean $\frac{1}{\mu_{A}}$. The state of a node is independent of the states of any other node in the network. The evolution of the states of a node may be represented as a Markov chain as shown in the Figure 1.

In sleep state the nodes do not perform idle sensing and transmit operations, while in active state the nodes perform sensing, receiving and transmitting operations. Sensor networks may be classified according to the operations performed during sleep state in the following manner [25]:

1. Networks with idle sleep: The nodes neither sense nor re- ceive packets from neighboring nodes when they are in sleep state.These networks are suitable in situations where the node density is very high or there exists a high degree of redundancy in the information sensed by nodes. Also the delay requirement of the data should be elastic or else multiple paths should exist between sensors and sink.

2. Networks that sense during sleep: The nodes continue the sensing operation during sleep state but can not receive data from neighboring sensors. These networks are suitable in situations where the level of redundancy in the information sensed by various sensors is low. Thus each sensor must continue sensing operation for the network to function properly. However the delay requirements must be elastic or multiple paths between source and destination must exist.

3. Networks that receive during sleep: The nodes may receive packets from neighboring sensors but they do not sense during sleep state. These networks are suitable in situations where delay requirements are not elastic and redundancy in the information collected by various sensors in high.

4. Networks that sense and receive during sleep: The nodes continue their sensing and receiving operations during sleep state. These networks are suitable in situations where redundancy is low and delay requirements are stringent.

The power consumed by the networks progressively increases as we move down the above list. Networks with idle sleep consume the least power while networks that sense and receive packets during sleep consume the most power. We consider various sleep states in order to investigate the energy-performance tradeoffs among the above-mentioned energy conserving schemes. However, it should be noted that designing MAC protocols that realize some of the above sleep schemes may be difficult, particularly designing protocols that receive during sleep (without idle listening) may be most challenging. However, the packet reception may be enabled by using out-of-band signaling [26] or by the use of long preambles preceding a packet transmission and small periods idle listening during sleep state [27].

MAC model: Two nodes are said to be interfering neighbors if they lie within a distance of $2 r(n)$ of each other. The transmission of a node would be successful if none of the interfering neighbors of the node transmits concurrently. Also, two nodes may successfully transmit at the same time if they are not interfering neighbors of each other. This definition of interfering neighbors is similar to that given in [18].

The random access MAC model is described as follows. Before transmitting each packet the nodes count down a random back-off timer. The duration of the timer is exponentially distributed with mean $1 / \xi$. As in IEEE 802.11, the timer of a node freezes each time an interfering neighbor starts transmitting a packet. When the back-off timer of a node expires, it starts transmitting the packet and the back-off timers of all its interfering neighbors are immediately frozen. The timers of the interfering neighbors are resumed as soon as the transmission of the packet is complete. The time required to transmit a packet from a node to its neighbor is $L / W+T_{o}$, where $T_{o}$ is the time required for the exchange of RTS, CTS and ACK packets. We assume that $T_{o}$ is negligible compared to $L / W$, so in our analysis we assume that the time required to transmit a packet is $L / W$. The model is mathematically tractable and at the same time captures the behavior of IEEE 802.11 MAC protocol.

The backoff timer of a node, that is waiting to transmit, is frozen as soon as it enters sleep state. In the networks where nodes cannot receive during sleep state, if the MAC transmission timer expires 


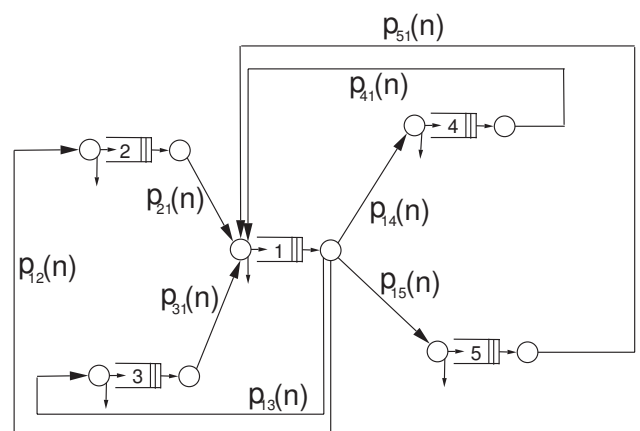

(a) Representation of multihop wireless sensor network as a queuing network.

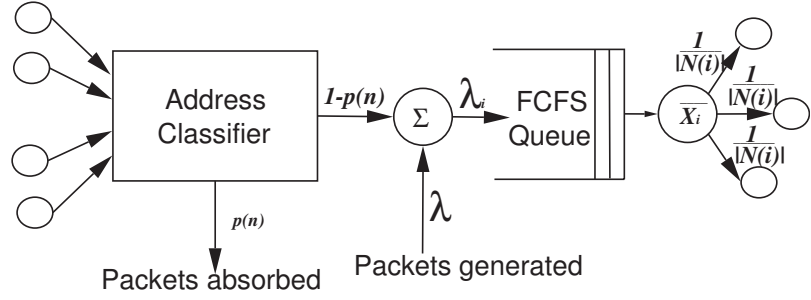

(b) Representation of a node of multihop wireless sensor network as a station in the queuing network.

Figure 2: Queuing network model for multihop wireless sensor networks.

while the intended receiver is sleeping then the node starts a new timer and makes another attempt to transmit the packet.

We assume that each node in the network has infinite buffers which means that no packets are dropped in the network. The packets are served by the nodes on first come first serve (FCFS) basis.

Queuing Network Model: Multihop wireless sensor networks can be modeled as a queuing network as shown in Figure 2(a). The stations of the queuing network correspond to the nodes of the wireless network. The forwarding probabilities in the queuing network, denoted by $p_{i j}$, correspond to the probability that a packet that is transmitted by node $i$ enters the node $j$ 's queue. Figure 2(b) shows a representation of a node in the sensor network as a station in the queuing network.

The end-to-end delay in a wireless network equals the sum of queuing and transmission delays at source and intermediate nodes. We will use the queuing network model shown in Figures 2(a) and 2(b) in order to mathematically analyze the end-to-end delay.

\subsection{Parameters of the queuing network model}

In this section we present expressions of the parameters of the queuing network model. For detailed derivations of these parameters please refer to [10].

LEMMA 1. The expected ${ }^{1}$ probability that a packet is forwarded from node $i$ to node $j$, denoted by $\overline{p_{i j}}(n)$, is

$$
\overline{p_{i j}}(n)= \begin{cases}\frac{1-p(n)}{n}\left(1-(1-A(n))^{n}\right) & i \neq j \\ 0 & i=j\end{cases}
$$

LEMma 2. The expected visit ratio of node $i$, denoted by $\overline{e_{i}}$, is given by

$$
\overline{e_{i}}=\frac{1}{(n+1) p(n)} \forall i
$$

when $(1-A(n))^{n} \rightarrow 0$, i.e. when $A(n)$ is chosen such that the network is connected with high probability.

LEMMA 3. The expected number of hops traversed by a packet between its source and destination, denoted by $\bar{s}$, equals $\frac{1}{p(n)}$.

\footnotetext{
${ }^{1}$ All expected values in this paper are the expectation over all packets and all possible network topologies.
}

\section{QUEUING ANALYSIS}

In this section we present the delay and capacity analysis for energy efficient networks with idle sleep. The analysis may be easily extended to the other sleep models. First we calculate preliminary quantities such as effective arrival rate, average interfering neighbors and the average number of active interfering neighbors that have a packet to transmit. Due to space constraints, the results are provided without proof. The detailed proofs are available in a technical report [28].

Lemma 4. Let $P_{A}$ denote the probability that a given node is in active state and $P_{S}$ denote the probability that the node is sleep state. Then

$$
\begin{aligned}
P_{A} & =\frac{\mu_{A}}{\mu_{A}+\mu_{S}} \\
P_{S} & =\frac{\mu_{S}}{\mu_{A}+\mu_{S}}
\end{aligned}
$$

LEMMA 5. The effective arrival rate as seen by a node in the sensor network is

$$
\lambda_{i}=\frac{\lambda P_{A}}{p(n)}
$$

LEMMA 6. Let $H_{i}$ denote the number of interfering neighbors of a node $i$. Then

$$
\begin{gathered}
E\left[H_{i}\right]=4 n A(n) \\
E\left[H_{i}^{2}\right]=4 n A(n)(1+4(n-1) A(n))
\end{gathered}
$$

where $A(n)=\pi \cdot r(n)^{2}$.

LEMMA 7. Let $M_{A i}$ denote the number of active interfering neighbors that have packets to send. Then under steady state,

$$
\begin{aligned}
E\left[M_{A i}\right]= & 4 \rho P_{A} n A(n) \\
E\left[M_{A i}^{2}\right]= & \rho^{2} P_{A}^{2} \cdot 4 n A(n)(1+4(n-1) A(n))+4 n A(n) \rho P_{A} \\
& \left(1-\rho P_{A}\right)
\end{aligned}
$$

Note that a single packet transmission may consist of several transmission attempts. These transmissions may fail because the intended receiver may be in sleep state. Each transmission commences when the transmission timer of a node expires. Each transmission attempt epoch consists of the time required for counting 
down the back-off timer and the time taken to transmit the packet. The time required to transmit the packet is constant and equals $L / W$ seconds. The time required for counting down the back-off timer will depend on the duration of the timer and the number of times it is frozen during the attempt epoch. The back-off timer may freeze due to two reason: (i) when an interfering neighbor starts transmitting, and (ii) when the node enters sleep state. The duration of the timer freeze induced by interfering neighbors equals $L / W$ seconds. The mean duration of the freeze induced due to the sleep state equals $1 / \mu_{A}$ seconds. Let $Z_{A i}$ and $W_{A i}$ denote the number of times the timer is frozen during a transmission attempt epoch at node $i$ due to interfering neighbors and sleep state respectively. Let $\chi_{i}$ denote the length of a transmission attempt epoch. In order to calculate $\chi_{i}$, we first need to calculate $Z_{A i}$ and $W_{A i}$. Also note that since the probability that the intended receiver is in active state when the timer expires $P_{A}$, the mean time required to serve a packet at node $i$ (denote by $\bar{X}_{i}$ ) equals $E\left[\chi_{i}\right] / P_{A}$. We now proceed to calculate $E\left[Z_{A i}\right], E\left[W_{A i}\right], E\left[\chi_{i}\right]$, and $X_{i}$. In order to simplify the analysis and make it tractable we assume that the number of awake nodes in the neighborhood of a given node do not change during the transmission of a packet. For $\mu_{S}<<\mu_{A}$ these assumption would be very accurate.

LEMMA 8. Let $Z_{A i}$ denote the number of times the timer of a node is frozen during a transmission attempt epoch due to interfering neighbors. Then under steady state,

$$
E\left[Z_{A i}\right]=4 \rho n A(n) P_{A}
$$

LEMMA 9. Let $W_{A i}$ denote the number of times the back off timer is frozen during a transmission attempt epoch because a node enters sleep state. Then under steady state,

$$
E\left[W_{A i}\right]=\frac{\mu_{S} \mu_{A}}{\mu_{S}+\mu_{A}} E\left[\chi_{i}\right]
$$

where $\chi_{i}$ is the length of a transmission attempt epoch.

THEOREM 1. Let $\chi_{i}$ denote the duration of transmission attempt epoch of a node. Under steady state

$$
\begin{gathered}
\overline{\chi_{i}}=E\left[\chi_{i}\right]=\frac{\frac{1}{\xi}+\frac{L}{W}}{P_{A}-4 n A(n) \lambda_{i} \frac{L}{W}} \\
\overline{\chi_{i}^{2}}=E\left[\chi_{i}^{2}\right]=\left(\overline{Z_{A i}^{2}}\left(1+2 \frac{\mu_{S}}{\mu_{A}}\right)+2 \overline{Z_{A i}}\left(1+\frac{\mu_{S}}{\mu_{A}}\right)+1\right) \frac{L^{2}}{W^{2}}+ \\
\left(1+4 \overline{M_{A i}} \frac{\mu_{S}}{\mu_{A}}+2 \overline{M_{A i}}+\frac{\mu_{S}}{\mu_{A}}\right) \frac{L}{W} \frac{2}{\xi}+\left(1+2 \frac{\mu_{S}}{\mu_{A}}\right) \frac{2}{\xi^{2}}+\frac{2}{\mu_{A}^{2}} \overline{W_{A i}^{2}}+ \\
2 \frac{L}{W} \frac{1}{\mu_{A}} \overline{W_{A i}}
\end{gathered}
$$

COROLlaRY 1. Let $X_{i}$ denote the time required to serve a packet by node $i$. Then $\overline{X_{i}}$, the mean service time, and $\overline{X_{i}^{2}}$, the second moment of service time, are given by

$$
\begin{gathered}
\overline{X_{i}}=E\left[X_{i}\right]=\frac{\left(\frac{1}{\xi}+\frac{L}{W}\right) \frac{1}{P_{A}}}{P_{A}-4 n A(n) \lambda_{i} \frac{L}{W}} \\
\overline{X_{i}^{2}}=E\left[X_{i}^{2}\right]=\frac{1}{P_{A}} \overline{\chi_{i}^{2}}+\left(\frac{2}{P_{A}^{2}}-\frac{2}{P_{A}}\right){\overline{\chi_{i}}}^{2}
\end{gathered}
$$

THEOREM 2. For the idle sleep model, the average end to end delay in an energy efficient multihop wireless network, denoted by $D(n)$, is given by

$$
D(n)=\frac{\rho_{i}}{\lambda P_{A} \cdot\left(1-\hat{\rho}_{i}\right)}
$$

where $\lambda_{i}, \overline{X_{i}}$ and $\hat{\rho}_{i}$ are given by (12), (21) and (6) respectively and $\rho=\lambda_{i} \overline{X_{i}}$.

\section{DISCUSSIONS}

In the previous subsection we presented a delay analysis for energy efficient sensor network in which nodes do not receive packets or sense information while in sleep state. In this section we present, without proof the mean service time for all the sleep scenarios discussed in Section 3.1. We also present the expressions for maximum achievable throughput for all sleep scenarios. All results presented in this section are accompanied by a brief discussion on interpretation and implication of the results.

\subsection{Average Service Time}

1. For the networks with idle sleep ${ }^{2}$, the average service, $\overline{X_{i}^{\overline{R S}}}$, is given by

$$
\overline{X_{i}^{\overline{R S}}}=\frac{\frac{1}{\xi}+\frac{L}{W}}{P_{A}^{2}-4 n A(n) \frac{\lambda}{p(n)} \frac{L}{W} P_{A}^{2}}
$$

Consider a scenario where there are only two nodes; one transmits to the other. The transmitter uses 802.11 and both transmitter and receiver periodically go into idle sleep state. In such a scenario there would be no contention for the channel and the service time would be $\frac{1 / \xi+L / W}{P_{A}^{2}}$. The second term in the denominator $\left(4 n A(n) \frac{\lambda}{p(n)} \frac{L}{W} P_{A}^{2}\right)$ is the result of contention with neighboring nodes, and is termed contention term. In [10], it is shown that for networks where nodes do not enter sleep state, the service time in absence of contention is $\frac{1}{\xi}+\frac{L}{W}$ and the contention term appearing in the denominator is $4 n A(n) \frac{\lambda}{p(n)} \frac{L}{W}$. So the non-contended service time is increased by factor of $1 / P_{A}^{2}$ while the contention term is decreased by a factor of $P_{A}^{2}$.

The reason for the quadratic increase in the non-contended service time is that it is increased due to two reasons. One reason of the increase is that the transmitter periodically decides to sleep and the second reason is that the receiver might be sleeping when the back-off timer of transmitter expires and it is ready to transmit. Each of these factors contribute a factor of $1 / P_{A}$ to the non-contended service time.

Similarly, there are two reasons that lead to the decrease of the contention term. One reason is that the probability that the nodes contending with the transmitter are active is $P_{A}$. Thus contention is reduced because some of the contending nodes may be sleeping. The second reason for decrease in contention is that the nodes produce data (or sense) only when they are active. Thus utilization factor of each node is reduced and so is contention. A factor of $P_{A}$ is contributed due to each reason and hence the contention term is reduced by factor $P_{A}^{2}$.

${ }^{2}$ Although this result has been worked out in previous subsection it presented here for the sake of completeness and elaborate discussion 
2. For the networks where nodes sense during sleep but do not receive packets from neighbors, the average service time is

$$
\overline{X_{i}^{\bar{R} S}}=\frac{\frac{1}{\xi}+\frac{L}{W}}{P_{A}^{2}-4 n A(n) \frac{\lambda}{p(n)} \frac{L}{W} P_{A}}
$$

In this case the service time in absence of contention is increased by a factor of $1 / P_{A}^{2}$. The reason for this decrease is the same as explained in the last section i.e. due to the periodic sleep of both transmitter and receiver. However the contention term is decreased by a factor of $P_{A}$ only. This decrease is because contending nodes are in sleep state with probability $P_{A}$. However since the nodes are producing packets even during sleep we do not see a quadratic decrease in contention term as observed in the case of idle sleep.

3. For networks where nodes receive packets from neighbors during sleep but do not perform sensing operation, the average service time is given by

$$
\overline{X_{i}^{R \bar{S}}}=\frac{\frac{1}{\xi}+\frac{L}{W}}{P_{A}-4 n A(n) \frac{\lambda}{p(n)} \frac{L}{W} P_{A}^{2}}
$$

In this case the service time in absence of contention is increased by a factor of $1 / P_{A}$ only. The factor is not quadratic because the increase in this case in caused only because of the sleep of transmitting node. The packet can be successfully transmitted by the transmitter even if receiver node is sleeping. The contention term is decreased by a factor $P_{A}^{2}$. The reason for this factor is same as that for idle sleep.

4. For networks where nodes receive packets from neighboring nodes and perform sensing operations in sleep state, the average service time is

$$
\overline{X_{i}^{R S}}=\frac{\frac{1}{\xi}+\frac{L}{W}}{P_{A}-4 n A(n) \frac{\lambda}{p(n)} \frac{L}{W} P_{A}}
$$

In this case the increase in service time without any contention is by a factor of $1 / P_{A}$. This is because nodes may receive successfully during sleep and increase in non-contended service time is only because transmitter may go into sleep mode during service epoch. The contention term is decreased by factor $P_{A}$. The decrease is because the probability that contending node is sleeping (and hence not contending) is $P_{A}$.

\subsection{Maximum Achievable Sensing Rate}

In this subsection we present the maximum rate at which the nodes may sense data for various sleep scenarios. These results are obtained by using the fact that the utilization ratio $\rho=\lambda_{i} \bar{X}_{i}$ should be less than 1 for delay to be finite.

1. For the networks with idle sleep, the maximum achievable throughput is

$$
\lambda_{\max }^{\overline{R S}}=\frac{p(n)}{\frac{1 / \xi+L / W}{P_{A}}+4 n A(n) \frac{L}{W}}
$$

The result may be interpreted in the following way. For the case of only one transmitter and one receiver, where both receiver and transmitter do not receive during sleep, the maximum achievable throughput would be $\frac{1}{(1 / \xi+L / W) / P_{A}^{2}}$. In a network with many nodes but single hop traffic, the contention term of $4 n A(n)(L / W) / P_{A}^{2}$ would be present. Because the neighboring nodes are active with probability $P_{A}$, the contention term is reduced to $4 n A(n)(L / W) / P_{A}$. Since the traffic is multi-hop and nodes do not sense while sleeping the maximum achievable throughput is given by

$\frac{p(n) / P_{A}}{(1 / \xi+L / W) / P_{A}^{2}+4 n A(n)(L / W) / P_{A}}$ which reduces to (28).

2. For the networks where nodes sense during sleep but do not receive packets from neighbors, the maximum achievable throughput is

$$
\lambda_{\max }^{\bar{R} S}=\frac{p(n)}{\frac{1 / \xi+L / W}{P_{A}^{2}}+4 n A(n) \frac{L}{W} \frac{1}{P_{A}}}
$$

The same explanation given in the case of idle sleep applies to this case except that because the nodes sense during sleep the $1 / P_{A}$ term does not appear in the numerator in the final expression as it does in the case of idle sleep.

3. For networks where nodes receive packets from neighbors during sleep but do not perform sensing operation, the maximum achievable throughput is

$$
\lambda_{\max }^{R \bar{S}}=\frac{p(n)}{1 / \xi+L / W+4 n A(n) \frac{L}{W} P_{A}}
$$

For this case the maximum achievable throughput in a twonode network would be $\frac{1}{(1 / \xi+L / W) / P_{A}}$. The term due to contention would be $\left(4 n A(n) \cdot P_{A}\right) / P_{A}=4 n A(n)$. The $P_{A}$ factor in numerator appears because contending nodes are active with probability $P_{A}$ while the $P_{A}$ factor in the denominator appears because the node itself is active only for $P_{A}$ fraction of time. Incorporating the fact that the traffic is multihop and that the nodes do not sense while sleeping we get (30).

4. For networks where nodes receive packets from neighboring nodes and perform sensing operations in sleep state, the maximum achievable throughput is

$$
\lambda_{\max }^{R S}=\frac{p(n)}{\frac{1 / \xi+L / W}{P_{A}}+4 n A(n) \frac{L}{W}}
$$

For this case the maximum achievable throughput for the two node network would be $\frac{1}{(1 / \xi+L / W) / P_{A}}$. The contention term would be $\left(4 n A(n) \cdot P_{A}\right) / P_{A}=4 n A(n)$. Since the traffic is multi-hop and nodes continue to sense while sleeping the maximum achievable throughput is (31).

Another related concept is the maximum achievable effective throughput of a node. The effective throughput indicates the rate at which a node generates packets. For the cases where nodes do not sense while in sleep state ( 1 and 3 ), the effective throughput equals the sensing rate times the probability of a node being in active state. Thus, the effective throughput only depends on the fact that whether nodes receive during sleep state or not. So for case where nodes do not receive while in sleep state the effective throughput is given by

$$
\lambda_{e f f}^{\bar{R}}=\frac{p(n)}{\frac{1 / \xi+L / W}{P_{A}^{2}}+4 n A(n) \frac{L}{W} \frac{1}{P_{A}}}
$$

On the other hand, for cases where nodes can receive packets in sleep state the maximum achievable effective throughput is given by

$$
\lambda_{e f f}^{R}=\lambda_{\max }^{R S}=\frac{p(n)}{\frac{1 / \xi+L / W}{P_{A}}+4 n A(n) \frac{L}{W}}
$$




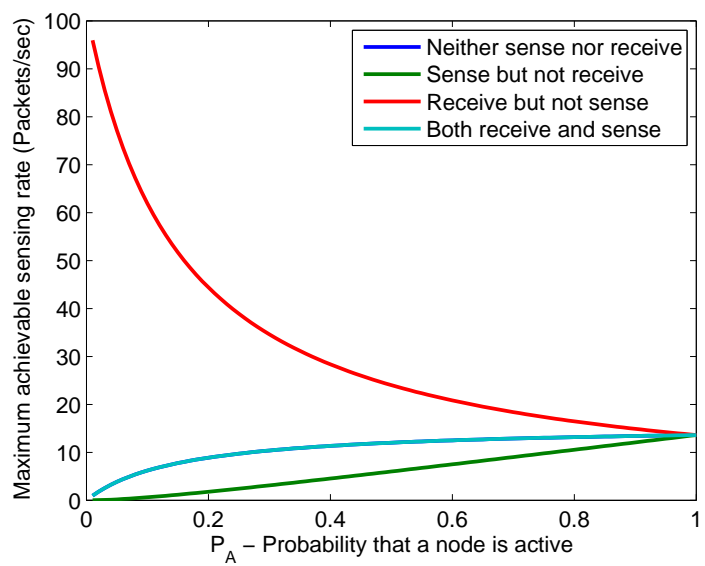

Figure 3: Maximum sensing rate versus probability that a node is active for various sleep scenarios. The sensing rate for scenario 1 and 4 overlap with each other.

We now present and discuss some numerical results of the above analysis. For these results we used the following network parameters: $n=500, \xi=10^{3}$ sec, $L=1000$ bits, $W=11 \mathrm{Mbps}$, $p(n)=\sqrt{\log n / n}$, and $r(n)=\sqrt{\log n / n}$. Figure 3 shows how the maximum achievable sensing rate varies with $P_{A}$ for all sleep scenarios. It is observed that, except for the scenario where nodes receive packets during sleep state (case 3 ) but do not generate any traffic, the sensing rate increases with increase in $P_{A}$. This trend can be explained in the following manner. In case 3 , the sensors do not generate any new data during the sleep state. As $P_{A}$ increases the effective rate of packet generation increases, which increases load on the network for a given sensing rate. Thus maximum achievable sensing rate decreases as $P_{A}$ increases. The maximum achievable sensing rate is the least for case 2 , where nodes continue to generate traffic during sleep state but do not receive packets. This is because the effective packet generation rate is high for a given sensing rate and the fact that nodes do not receive packets during sleep state increases the service time. This leads to lowest maximum sensing rate. The maximum sensing rate for case 1 and 4 overlap. In case 1 nodes neither generate traffic nor receive packets during the sleep state. In case 4 the nodes continue to generate traffic during sleep state but it is compensated by their ability to receive packets during sleep state. In cases 1 and 2 the sensing rate increases with increase in $P_{A}$ since increase in $P_{A}$ implies the nodes are ready to receive and transmit packet for more fraction of time. In case 3 , the increase in sensing sensing with $P_{A}$ is only due to the ability of nodes to transmit for more fraction of time with increase in $P_{A}$. In case 3 also the fraction of time that the nodes are able to transmit increases with increase in $P_{A}$, it is outweighed by the fact that the generate more traffic with increase in $P_{A}$.

Figure 4 shows how the effective throughput varies with $P_{A}$. We observe that for both the cases where the nodes may receive or not receive during the sleep state, the maximum achievable throughput increases with increase in $P_{A}$. This is because increase in $P_{A}$ allows nodes to be able to transmit packets for a larger fraction of time and hence decreasing the service time. This result also implies that the maximum achievable throughput of an energy efficient sensor network is always less than that of a normal ad hoc network. In other words, the reduction in channel contention due to the nodes periodically switching over to sleep state is outweighed

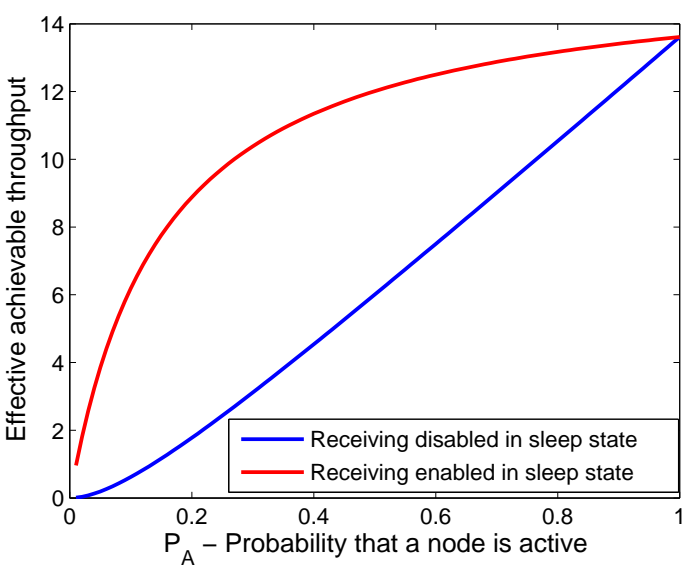

Figure 4: Maximum achievable effective throughput versus probability that a node is active. No matter whether nodes receive data during sleep or not, the maximum achievable throughput increases with active probability.

by the longer delays incurred due to the nodes not being able to transmit during sleep state. Also another observation form 4 is that the effective throughput is higher when the nodes are able to receive packets during sleep state. In fact, the difference is very significant when $P_{A}$ is small. However ability to receive the packets at all times increases the energy spent during the sleep state and hence lowers the network lifetime. In fact in the currently existing transreceivers and protocols the ability to receive packets during sleep state may lead to little or no energy savings. Thus from the point of view of conserving energy it is desirable that the nodes do not perform receive operations during the sleep state. In other words, the results highlight the importance of developing efficient mechanisms which enable the receivers to wake-up just-in-time (possibly for a short period of times) to receive packets from their neighbors, or to develop efficient mechanisms to synchronize sleep schedule of neighbors.

\section{SUMMARY}

In this paper we studied the impact of strategies to conserve energy on the delay and capacity of multihop wireless networks. The energy conservation strategies considered in this paper are periodic switch to a low energy sleep state. The nodes cannot transmit packets in a sleep state, however in some cases nodes may be allowed to receive or keep on generating data while in sleep state. We use a queuing theoretic approach in order to evaluate the end-to-end delay and maximum achievable effective throughput. It is observed that no matter what sleep strategy is employed, the effective throughput of energy efficient networks is less than that of ad hoc networks with similar network parameters that do not employ any energy conservation mechanism. This is because the reduction in channel contention due to the nodes switching to sleep state is outweighed by the increased delay introduced due to nodes not being able to transmit or receive packets during sleep state. Numerical results are presented which highlight the importance of developing correlated sleep schedule or mechanisms that allow nodes to efficiently receive packets in sleep state. 


\section{Acknowledgment}

This work was funded by the National Science Foundation under grant number CNS-546402.

\section{REFERENCES}

[1] J.-H. Chang and L. Tassiulas, "Energy conserving routing in wireless ad-hoc networks," in INFOCOM 2000. Nineteenth Annual Joint Conference of the IEEE Computer and Communications Societies. Proceedings. IEEE, vol. 1, Tel Aviv, Israel, 2000, pp. 22-31.

[2] S. Singh, M. Woo, and C. S. Raghavendra, "Power-aware routing in mobile ad hoc networks." in MOBICOM, 1998, pp. 181-190.

[3] B. Prabhakar, E. Uysal Biyikoglu, and A. El Gamal, "Energy-efficient transmission over a wireless link via lazy packet scheduling," in INFOCOM 2001. Twentieth Annual Joint Conference of the IEEE Computer and Communications Societies. Proceedings. IEEE, vol. 1, Anchorage, AK, USA, 2001, pp. 386-394.

[4] A. El Gamal, C. Nair, B. Prabhakar, E. Uysal-Biyikoglu, and S. Zahedi, "Energy-efficient scheduling of packet transmissions over wireless networks," INFOCOM 2002. Twenty-First Annual Joint Conference of the IEEE Computer and Communications Societies. Proceedings. IEEE, vol. 3, pp. 1773-1782, 2002.

[5] Y. Yu, B. Krishnamachari, and V. K. Prasanna, "Energy-latency tradeoffs for data gathering in wireless sensor networks," INFOCOM 2004. Twenty-third AnnualJoint Conference of the IEEE Computer and Communications Societies, vol. 1, Mar. 2004.

[6] B. Krishnamachari, D. Estrin, and S. Wicker, "Impact of data aggregation in wireless sensor networks," in Proceedings of International Workshop of Distributed Event Based Systems (DEBs), 2002. [Online]. Available: citeseer.ist.psu.edu/597882.html

[7] W. Ye, J. Heidemann, and D. Estrin, "An energy efficient MAC protocol for wireless sensor networks," in Proceedings of IEEE INFOCOM (INFOCOM'02). IEEE, June 2002.

[8] S. Kumar, T. H. Lai, and J. Balogh, "On K-Coverage in a Mostly Sleeping Sensor Network," in Proceedings of the 10th annual international conference on Mobile computing and networking. ACM Press, 2004, pp. 144-158.

[9] J. van Greunen, D. Petrovic, A. Bonivento, J. Rabaey, K. Ramchandran, and A. Sangiovanni-Vincentelli, "Adaptive Sleep Discipline for Energy Conservation and Robustness in Dense Sensor Networks," in Proceeding of the IEEE International Conference on Communication, 2004.

[10] N. Bisnik and A. Abouzeid, "Queuing network models for delay analysis of multihop wireless ad hoc networks," in Proceedings of International Wireless Communications and Mobile Computing Conference (IWCMC'06), 2006.

[11] S. Ramakrishnan, H. Huang, M. Balakrishnan, and J. Mullen, "Impact of sleep in a wireless sensor MAC protocol," 2004. VTC2004-Fall. 2004 IEEE 60th Vehicular Technology Conference, vol. 7, pp. 4621-4624, Sept. 2004.

[12] O. Dousse, P. Mannersalo, and P. Thiran, "Latency of wireless sensor networks with uncoordinated power saving mechanisms," in MobiHoc '04: Proceedings of the 5th ACM international symposium on Mobile ad hoc networking and computing. New York, NY, USA: ACM Press, 2004, pp. $109-120$
[13] L. Stabellini and A. Proutiere, "Evaluating delay and energy in sensor networks with sporadic and correlated traffic," in AdHoc'07, May 2007.

[14] G. Lu, B. Krishnamachari, and C. S. Raghavendra, "An adaptive energy-efficient and low-latency MAC for data gathering in wireless sensor networks," in Parallel and Distributed Processing Symposium, 2004. Proceedings. 18th International, Apr. 2004.

[15] G. Lu, N. Sadagopan, B. Krishnamachari, and A. Goel, "Delay efficient sleep scheduling in wireless sensor networks," INFOCOM 2005. 24th Annual Joint Conference of the IEEE Computer and Communications Societies. Proceedings IEEE, vol. 4, pp. 2470-2481, Mar. 2005.

[16] Q. Cao, T. Abdelzaher, T. He, and J. Stankovic, "Towards optimal sleep scheduling in sensor networks for rare-event detection," in IPSN '05: Proceedings of the 4th international symposium on Information processing in sensor networks. Piscataway, NJ, USA: IEEE Press, 2005, p. 4.

[17] G. Bolch, S. Greiner, H. de Meer, and K. S. Trivedi, Queuing Networks and Markov Chains. John Wiley and Sons, 1998, ch. 10, pp. 423-430.

[18] P. Gupta and P. R. Kumar, "Capacity of wireless networks," IEEE Trans. on Information Theory, pp. 388-404, March 2000. [Online]. Available: citeseer.ist.psu.edu/gupta99capacity.html

[19] S. D. Servetto and G. Barrenechea, "Constrained random walks on random graphs: routing algorithms for large scale wireless sensor networks," in WSNA '02: Proceedings of the 1st ACM international workshop on Wireless sensor networks and applications. New York, NY, USA: ACM Press, 2002, pp. 12-21.

[20] H. Tian, H. Shen, and T. Matsuzawa, "Random walk routing for wireless sensor networks," in PDCAT '05: Proceedings of the Sixth International Conference on Parallel and Distributed Computing Applications and Technologies. Washington, DC, USA: IEEE Computer Society, 2005, pp. 196-200.

[21] S. Shakkottai, "Asymptotics of query strategies over a sensor network." in INFOCOM, 2004.

[22] M. Zuniga, C. Avin, and B. Krishnamachari, "Using heterogeneity to enhance random walk-based queries," USC Computer Engineering Technical Report CENG-2006-8, August 2006.

[23] C. Avin and B. Krishnamachari, "The power of choice in random walks: An empirical study," in In 9th ACM/IEEE International Symposium on Modeling, Analysis and Simulation of Wireless and Mobile Systems (MSWiM), October 2006.

[24] N. Sadagopan, B. Krishnamachari, and A. Helmy, "Active query forwarding in sensor networks (ACQUIRE)," Elsevier Ad Hoc Networks, vol. 3, no. 1, pp. 91-113, January 2005.

[25] A. Sinha and A. Chandrakasan, "Dynamic power management in wireless sensor networks," IEEE Design \& Test of Computers, vol. 18, no. 2, pp. 62-74, Mar./Apr. 2001.

[26] S. Singh and C. S. Raghavendra, "PAMAS-power aware multi-access protocol with signalling for ad hoc networks," SIGCOMM Comput. Commun. Rev., vol. 28, no. 3, pp. 5-26, 1998.

[27] J. Polastre, J. Hill, and D. E. Culler, "Versatile low power media access for wireless sensor networks." in SynSys, 2004, pp. 95-107.

[28] N. Bisnik and A. A. Abouzeid, "Delay and capacity in energy 
efficient sensor networks," Technical Report, ECSE Dept.,

RPI. Available at

http://www.ecse.rpi.edu/homepages/abouzeid/energyEfficient.pdf. 